This item was submitted to Loughborough's Research Repository by the author.

Items in Figshare are protected by copyright, with all rights reserved, unless otherwise indicated.

\title{
Interplay of sketches and mental imagery in the design ideation stage of novice designers
}

PLEASE CITE THE PUBLISHED VERSION

https://doi.org/10.1080/14606925.2018.1395655

\section{PUBLISHER}

(C) Taylor \& Francis

\section{VERSION}

AM (Accepted Manuscript)

\section{PUBLISHER STATEMENT}

This is an Accepted Manuscript of an article published by Taylor \& Francis in The Design Journal on 16 November 2017, available online: http://www.tandfonline.com/10.1080/14606925.2018.1395655.

\section{LICENCE}

CC BY-NC-ND 4.0

\section{REPOSITORY RECORD}

Tedjosaputro, Mia A., Yi-Teng Shih, Chantelle Niblock, and Patrick Pradel. 2019. "Interplay of Sketches and Mental Imagery in the Design Ideation Stage of Novice Designers". figshare. https://hdl.handle.net/2134/28262. 


\title{
Interplay of Sketches and Mental Imagery in the Design Ideation Stage of Novice Designers
}

\author{
This paper seeks to understand the interplay between the acts of (pen-and-paper \\ based) sketching and the use of mental imagery. A protocol study of four novice \\ designers was conducted in two different environments: with access to sketches \\ only; and blindfolded and delayed sketches. In total there are eight forty-five \\ minute design sessions. This study confirms that sketches and mental imagery \\ support ideation stages in unique ways and both are equally germane. Identified \\ interplaying roles when only sketches are utilised are: supporting, co-evolving \\ and reflecting roles. In addition, interplay can happen at the exact moment or \\ distantly; and the order of an interplay depends on the designers' strategy. In \\ sessions where sketches are for externalising design proposals only at the end, \\ identified roles are: generative, and provocative to idea exploration. It is also \\ observed that gesture does not compensate for the absence of external \\ representation; although it conveys important design messages.
}

Keywords: sketching; mental imagery; design ideation; protocol analysis; Linkography, Function-Behaviour-Structure

\section{Introduction}

The importance of exploring the relationship between external and internal representations lies in the fact that it is generally assumed. This paper aims to provide empirical evidence of how these simultaneous actions affect each other and provides fresh perspectives on this particular concept of simultaneity. It focuses only on the design ideation stage, when designers immediately become aware of design briefs. The scope of the ideation stage here entails producing possible design solutions. With the proliferation of studies related to expert designers, this study seeks to provide a revealing viewpoint of less experienced designers. With less exposure to design precedents, previously generated similar ideas and smaller mental image banks, it is hoped that it will reveal different ways to generate and manipulate ideas. 
The general concept of 'interplay' is generally understood as: more than one matter, action or reaction and ways two or more matters affect each other. The question the authors would like to answer is, 'What are the interplaying roles of sketches and mental imagery in the idea generation stage?' The outcome is explored through two hypotheses:

- H1- In sessions which access to external representations are limited, mental images are recalled to be manipulated further during EXT sessions.

- H2- With access to external representations, the interplay between sketches and mental imagery vary in terms of sequence and reciprocity, depending on designers' strategy and intention.

The posited research question and hypotheses outline the paper as they are used to categorise literature, data exploration and findings. Abbreviations which are used throughout the paper will then be enumerated. There are two design session environments: firstly, SK (Sketching) session which is a forty-five minute pen-andpaper on special dotted paper session. Secondly, MI (Mental Imagery) session which consists of thirty-five minutes of BF (Blindfolded) session and ten minutes of EXT (Externalisation) session. Two design briefs are abbreviated as: FMS (Flexible Meeting Space) and HFS (Hybrid Furniture System). The study looked at two three-dimensional design domains namely: PDM (Product Design and Manufacturing) and architecture. Verbal data is encoded using the FBS (Function-Behaviour-Structure) ontology and coding scheme.

\section{Relevant literature}

In creative discovery, the use of design sketches has been long accepted as a fundamental aspect of designing and has thoroughly been researched. Early design 
sketches not only inhibit the physical properties, but also cognitive processes simultaneously happen when a designer produces a drawing. Simultaneous processes between external representations and internal representations while a designer is engaged in a design process have been mentioned but are not adequately studied. Sketches are half-percept mental image that amplify the mind's capacity to make descriptive-to-depictive translation (Fish and Scrivener 1990), and one of sketching's cognitive advantages is that it supports feedback loops between external and internal representations (Goldschmidt 2013). In addition, Kavakli and Gero (2002) are also in accord with this view of interplay.

The unique kind of interactivity between sketches and mental imagery is a point of departure for this study. This paper intends to demystify this esoteric nature of the design ideation stage, specifically at the utilisation of sketches and mental imagery.

Free-hand drawings produced at the beginning of the design stage, after a task is given, usually executed rapidly and sometimes known as idea sketches; are what were subscribed to. 'Mental imagery occurs when perceptual information is accessed from memory, giving rise to the experience of "seeing with the mind's eye", "hearing with the mind's ear" and so on' (Kosslyn, Ganis, and Thompson 2006). It is not only recalling previously perceived objects, but also combining and modifying stored perceptual information (ibid). Quasi-pictorial theory is what the authors ascribe to. It is based on Kosslyn's publication in 2006 (Kosslyn, Thompson, and Ganis 2006), which replaced the previous version in 1980 . The key property is that it uses space in representational media to represent space in the world.

A subset of findings of external and internal representations studies, building upon previous studies is illustrated below. Table 1 shows the possibility that sketches and mental imagery strategies may be deployed either in isolation (findings number 2 
and 3) or in concert (findings number 1 and 4). This paper focuses on the latter discussion.

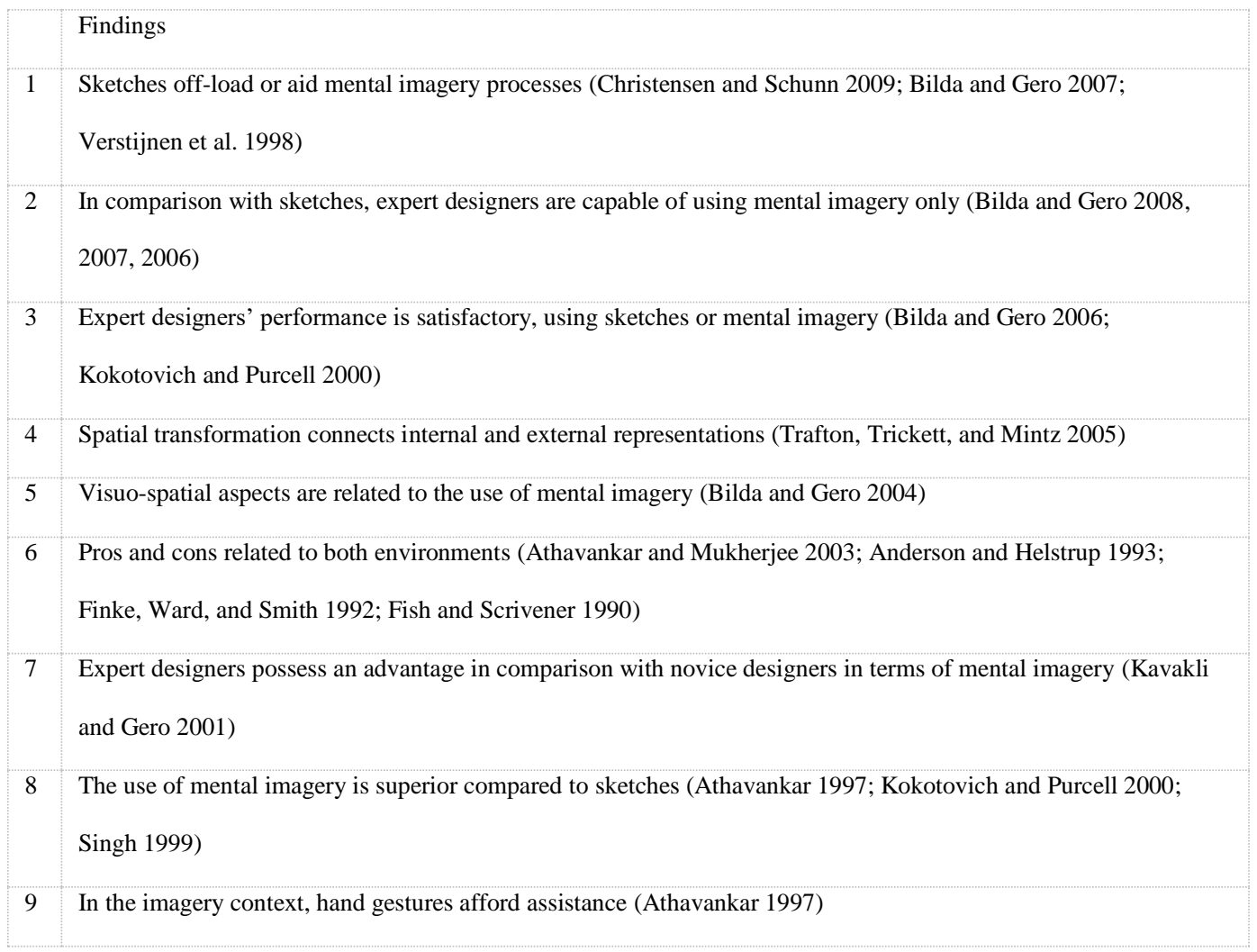

Table 1. Findings of previous studies in external and internal representations

A basic sense of concept of simultaneity is utilised to understand the interplaying role. Simultaneity is defined as the 'temporal coincidence of events' (Jammer 2006). It can also be spatially separated events with synchronised clocks (ibid). The principle of coexistence by Immanuel Kant in the classical physics era explains that event $e_{1}$ is simultaneous with event $e_{2}$ if and only they both share mutual interactions. The notion provides a plausible and different way to understand interplay. 'all substances, so far as they can be perceived as coexisting in space, are always affecting each other reciprocally' or are 'in thoroughgoing reciprocal interaction'; and in perception of simultaneous things the order is arbitrary (ibid). 


\section{Protocol study and data}

The basic assumption of protocol studies to which the authors subscribe: it is possible to instruct participants to verbalise thoughts while engaging in a task without altering the sequence and content of thoughts. Their verbal stream functions effectively as a "dump" of the contents of working memory. Ericsson and Simon (1993) explain it is necessary to keep verbalisation in Level 1 and 2 (no additional information from long term memory is heeded) and avoiding Level 3 (involving describing and explaining). In design studies, the think-aloud method has been a prominent design method. The protocol gives information about a designer's reasoning strategy which otherwise could not be obtained from the design proposals. It allows design researchers to understand strategies and knowledge used by designers.

Aiming to identify the interplay phenomenon without overarching generalisation, a controlled investigation setting was considered- a contrived setting with two controlled independent variables, the SK and MI design conditions.

Overall, there are five steps to obtain protocol data. Firstly, four novice designers participated on a voluntary basis, being final year design students of: architecture (abbreviated as P1 and P2) and product design and manufacturing (P3 and P4) programs in an overseas campus of a British university. The study has been reviewed according to the university's code of research conduct and research ethics, and consent was obtained from all participants. They are non-native English speakers. Given the choice to conduct the sessions in their mother tongue, all participants chose to do the sessions in English, the language of instruction of the university. Each designer underwent two design sessions (refer to Table 2); with a minimum one-month time gap between. There were eight sessions in total. The arrangement is modified from a previous study (Bilda, Gero, and Purcell 2006). 


\begin{tabular}{|c|c|c|c|c|}
\hline Sessions & Sub-sessions & About & Duration & Brief \\
\hline \multirow[t]{2}{*}{$\begin{array}{l}\text { S1-MI (Mental } \\
\text { Imagery) }\end{array}$} & $\begin{array}{l}\text { S1.1- BF } \\
\text { (Blindfolded) }\end{array}$ & $\begin{array}{l}\text { No visual access, only mental images } \\
\text { while thinking out loud. }\end{array}$ & 35 mins & FMS \\
\hline & $\begin{array}{l}\text { S1.2- EXT } \\
\text { (Externalisation) }\end{array}$ & $\begin{array}{l}\text { Externalising final design proposal } \\
\text { generated during EXT session, while } \\
\text { thinking out loud. }\end{array}$ & $10 \mathrm{mins}$ & \\
\hline $\begin{array}{l}\text { S2-SK } \\
\text { (Sketching) }\end{array}$ & - & $\begin{array}{l}\text { Full access to sketches using a smartpen } \\
\text { and special paper while thinking out } \\
\text { loud. }\end{array}$ & 45 mins & HFS \\
\hline
\end{tabular}

Table 2. Details of sessions

The two multifunctional design tasks were used as a middle ground between two different design domains. The design briefs consisted of: context of design, the task, and design solution suggested format. The suggested outcomes provide a guideline according to which any produced design ideas, solutions and final solutions have to be presented. One of the two design briefs can be found in Figure 1. The two design tasks were:

- Flexible Meeting Space (FMS), to design a convertible space for a creative industry company in an open plan office, less than 100sqm footprint.

- Hybrid Furniture System (HFS), to design a hybrid system of sitting space and dining set-up for adults and toddlers with maximum footprint of $3 \mathrm{mx} 3 \mathrm{~m}$.

The importance of blindfolds lies in eliminating visual cues and restricting visual or sensory modality. Also it gave no visual feedback, to ensure that designers rely on their memory only, as observed by Athavankar (1997) and Bilda, Gero, and Purcell (2006). During an interview of the first pilot study in 2014 which aimed to test out methodological issues of the study, one participant posited the idea of closing her eyes 
which made her realise how she could do more when it facilitated flashes of ideas.

\section{DESIGN BRIEF: \\ HYBRID FURNITURE SYSTEM FOR ADULTS AND TODDLERS}

You have understood the instructions given by the researcher, here is the task:

To design a hybrid furniture system for adults and children (toddlers). The flexible furniture serves as sitting space with a table, dining set-up for two adults; and reading and learning spaces for a child aged 4 years old. Maximum footprint is $3 \mathrm{~m} \times 3 \mathrm{~m}$. Please consider different requirements for adults and children. Also kindly describe the possible set-up options.

Minimum expected outcomes: plans with dimensions, elevations (side views) with dimensions and $3 \mathrm{~d}$ views. The rest of the drawings are subject to how you convey the design message.

The brief is open for interpretation for unspecified matters. Please only use the provided papers and ask experimenter for clarification if necessary.

Figure 1. HFS design brief

Secondly, three to four audio/ videos (front, top, side and a replayed pencast from a smartpen to capture the sketching process) were used to complement the transcription. The think-aloud method was used; a method in which designers verbalise their thoughts while designing. Participants were encouraged to verbalise thoughts as if they were talking to themselves.

Thirdly, the transcription stage. Due to the nature of the study, an exact reproduction of spoken words is necessary. Transcription was stored and managed digitally in CAQDAS (Computer Assisted Qualitative Data Analysis) software. In total, there are 360 minutes of verbal data derived from think-aloud sessions.

Fourthly, the segmentation step. For analysis, the verbal data was parsed into smaller chunks of activities. Protocol data (transcription) was segmented based on a design move, representing a singular design intention.

Fifthly, the encoding step. A pre-defined coding scheme was used, based on FBS (Function-Behavior-Structure) ontology (Gero 1990; Gero and Mc Neill 1998; Gero and Kannengiesser 2004). Each segment was coded based on design issues. 
Among other established coding schemes to capture designers' cognitive strategies, FBS coding scheme has been used for multidisciplinary design domains and it is deemed to be suitable for this study.

The ontology represents design process in terms of six design issues: ' $F$ 'function, Behaviour ('Be'-expected behaviour and 'Bs'-behaviour derived from structure) and ' $S$ '-structure and external classes: 'D'- documentation and 'R'requirement, which then forms eight FBS design processes in relation to the issues. In the first process, formulation transforms design requirements expressed in function into behaviour, expecting to enable the function $(\mathrm{F} \rightarrow \mathrm{Be})$ is seen as a transformation of design intentions from ' $\mathrm{R}$ ' to ' $\mathrm{Be}$ ' and expressed in terms of ' $\mathrm{F}$ '. Synthesis $(\mathrm{Be} \rightarrow \mathrm{S})$ is the transformation of 'Be' to ' $\mathrm{S}$ '. Analysis $(\mathrm{S} \rightarrow \mathrm{Bs})$ is the derivation of 'Bs' from the synthesised ' $\mathrm{S}$ '. Evaluation (Be $\leftrightarrow \mathrm{Bs}$ ) is a comparison processes between 'Bs' and 'Be' to decide whether design solution is to be accepted. Documentation $(\mathrm{S} \rightarrow \mathrm{D})$ is the production of 'D'. Reformulation type I $(\mathrm{S} \rightarrow \mathrm{S})$ addresses changes in design state space in terms of structure variables; Reformulation type II $(\mathrm{S} \rightarrow \mathrm{Be})$ in terms of expected behaviour variables; and Reformulation type III $(\mathrm{S} \rightarrow \mathrm{F})$ in terms of function variables.

\section{Method of analysis}

Linkography is a notation system used to analyse design processes that focuses on links between design moves (Goldschmidt 2014). A design move is presented as one segment derived from the verbal data, the smallest semantic units. Links between moves are decided by judges with educated common sense, by asking "is there a link?" for every pair of moves (ibid). The typical result is called a linkograph which displays a design activities network. A thorough explanation of linkography is beyond the scope of this paper, however, related concepts such as: forelinks, backlinks, critical moves and link 
index will be iterated. Designers move between cycles of ideation (looking forwardforelinks) and evaluation (looking backward- backlinks). Moves with large numbers of links are defined as critical moves (CMs). Link index factors is the length of the linkograph, the proportion of links in network. It is defined as the ratio between number of links and number of moves.

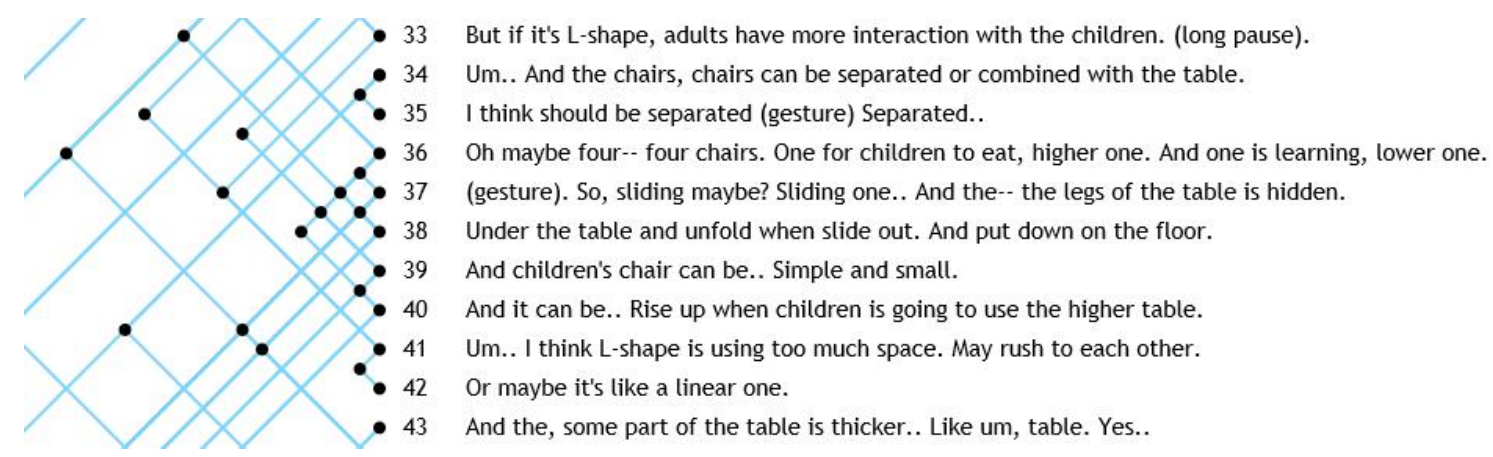

Figure 2. Excerpt of P1_MI's utterances and links

Figure 2 shows an excerpt of P1's mental imagery session, generated by an automated script developed by the authors for the purpose of data exploration. Links between each utterance are defined firstly with keywords (for instance in utterance \#33, 'L-shape'). The keyword is mentioned again in utterance \#41 as P1 justified the shape, and next a link between \#33 and \#41 is established) illustrates the judge's decisions of continuous idea development between previous utterance (for instance \#34 and \#35) or previously mentioned/ developed ideas within the session. During defining the links, when a new idea is posited (for example \#36 about four chairs), the utterance is noted as an important utterance.

Compared to other methods, Linkography provides a useful tool to understand how designers develop ideas overall. Combined with FBS coding scheme for more specific activities, the researchers intend to explore the flexibility of shifting between macro scale and micro scale at any given designing time. LINKODER (previously 
LINKOgrapher) is used, a software tool to analyse coded segments and linkographs developed by Pourmohamadi Mahounaki and Gero (2011).

\section{Results}

\begin{tabular}{|l|lll|lll|}
\hline Participants & Design & Total & Non-FBS & Total & Link & No of \\
\hline & sessions & segments & segments & Links & Index & page \\
\hline P1 & P1_MI & 199 & $4(2 \%)$ & 309 & 1.55 & 3 \\
\hline P2 & P1_SK & 186 & $3(1 \%)$ & 374 & 2.01 & 5 \\
\hline P3 & P2_MI & 229 & $11(4 \%)$ & 406 & 1.77 & 1 \\
\hline & P2_SK & 183 & $8(4 \%)$ & 291 & 1.59 & 2 \\
\hline P4 & P3_MI & 182 & $7(3 \%)$ & 286 & 1.56 & 2 \\
\hline & P3_SK & 111 & $3(2.7 \%)$ & 197 & 1.08 & 8 \\
\hline & P4_MI & 200 & $5(2 \%)$ & 267 & 1.34 & 1 \\
\hline
\end{tabular}

Table 3. General statistics of eight design sessions

Table 3 illustrates how four participants and eight design sessions are labelled and will be used for the remainder of this paper. Non-FBS segments are void utterances which do not convey any design intention. In general, all four mental imagery sessions produced more segments in comparison with sketching sessions. In terms of individual sessions; session P2_MI which has the highest number of total segments, provides the highest total number of links. However, the second highest number of total segments (session P4_MI) does not yield the second highest number of total links.

\begin{tabular}{|c|c|c|c|c|c|c|c|c|}
\hline Issue Distribution (\%) & P1_MI & P1_SK & P2_MI & P2_SK & P3_MI & P3_SK & P4_MI & P4_SK \\
\hline R- Requirements & 4.1 & 13.7 & 11.5 & 4.6 & 8.0 & 0.9 & 11.3 & 15.9 \\
\hline F- Function & 10.8 & 4.9 & 22 & 23.4 & 9.1 & 13.9 & 7.7 & 8.3 \\
\hline Be- Expected Behaviour & 14.4 & 16.4 & 18.8 & 9.1 & 17.0 & 23.1 & 24.1 & 15.2 \\
\hline Bs- Behaviour derived from structure & 26.2 & 14.2 & 16.5 & 20.0 & 14.2 & 14.8 & 23.6 & 31.1 \\
\hline S- Structure & 44.1 & 48.1 & 21.1 & 31.4 & 48.3 & 46.3 & 27.2 & 26.5 \\
\hline D- Documents or design descriptions & 0.5 & 2.7 & 10.1 & 11.4 & 3.4 & 0.9 & 6.2 & 3.0 \\
\hline
\end{tabular}

Table 4. Distribution of design issues 
Table 4 provides percentage of the coded activities. The highest number for each participant is highlighted in bold. S- structure variable which describe components of an object and their relationship dominates in six design sessions, except sessions P2_MI and P4_SK which ranked second highest.

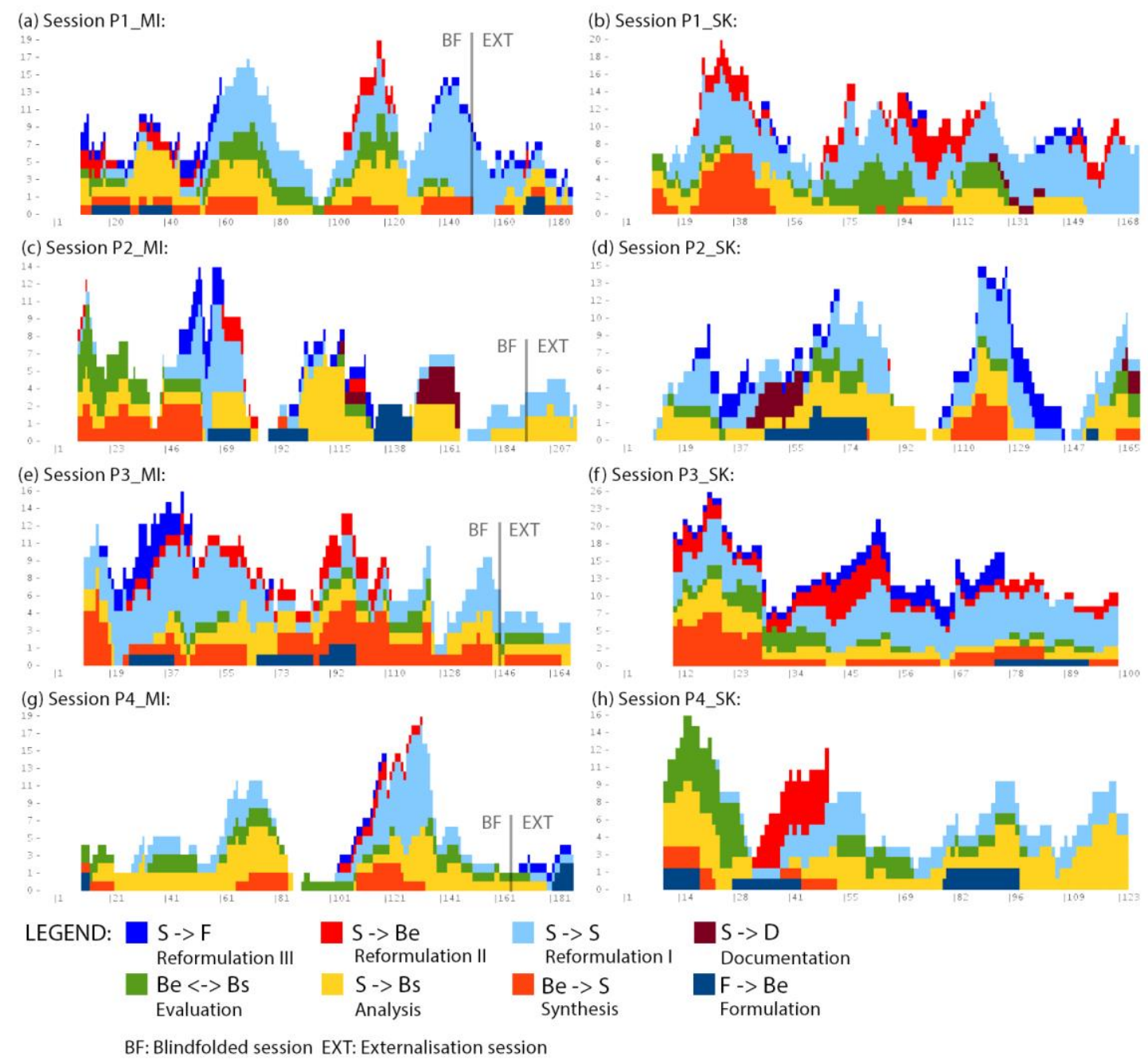

Figure 3. Semantic dynamic processes of eight design sessions

Figure 3 shows semantic dynamic processes distribution over eight design sessions. Most large peaks are related to Reformulation I, II or III; except P4_SK in Figure 3(h). In all MI sessions, particularly in BF (Blindfolded) sessions there are two distinguished parts of BF session splitting the sub-session into two chunks, mostly in the middle of the sessions. However, in SK sessions, more erratic distributions of peaks appear throughout the session. 
Each design session is marked by four phases, refer to Table 5. Figure 4 elicits eight linkographs produced using LINKODER and edited.

\begin{tabular}{|l:l|l|}
\hline Phases & Duration & Remarks \\
\hline Phase 1 & 15 minutes & - \\
\hline Phase 2 & 10 minutes & - \\
\hdashline Phase 3 & 10 minutes & - \\
\hline Phase 4 & 10 minutes & EXT sessions (during MI sessions only) \\
\hline
\end{tabular}

Table 5. Design phases

(a) Session P1_MI:

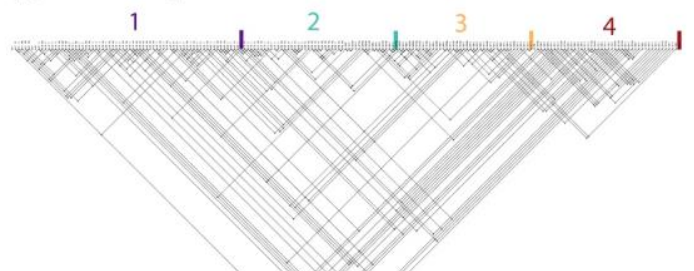

(c) Session P2_MI:

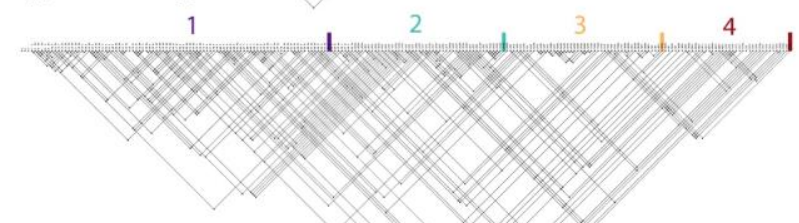

(e) Session P3_MI:

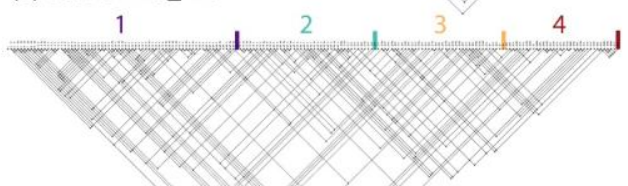

(g) Session P4_MI:

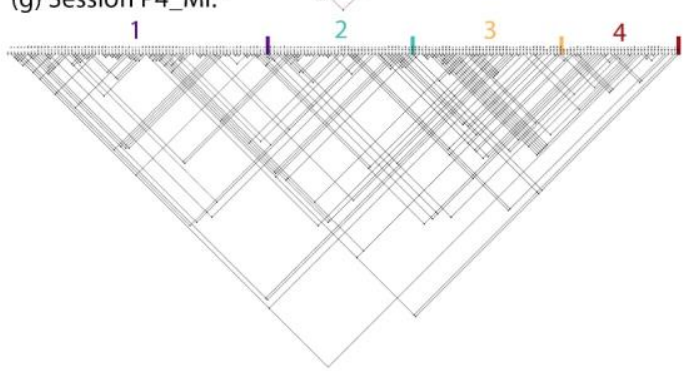

(b) Session P1_SK:

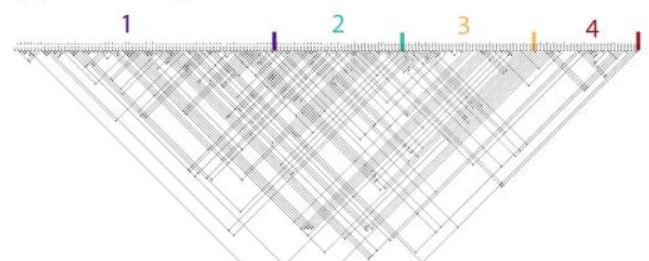

(d) Session P2_SK:

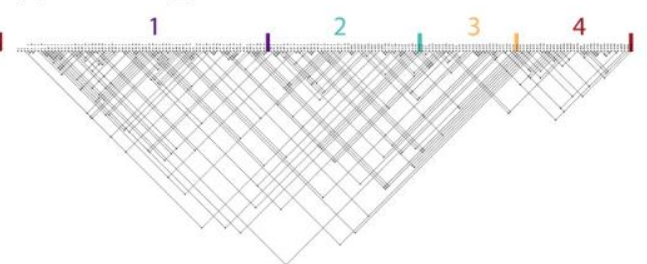

(f) Session P3_SK:

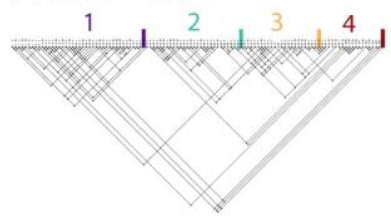

(h) Session P4_SK:

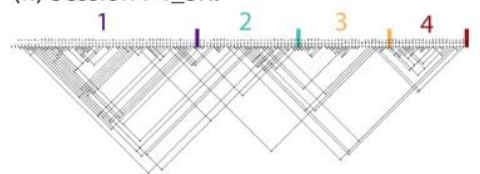

Figure 4. Linkographs of eight sessions with labelled phases 


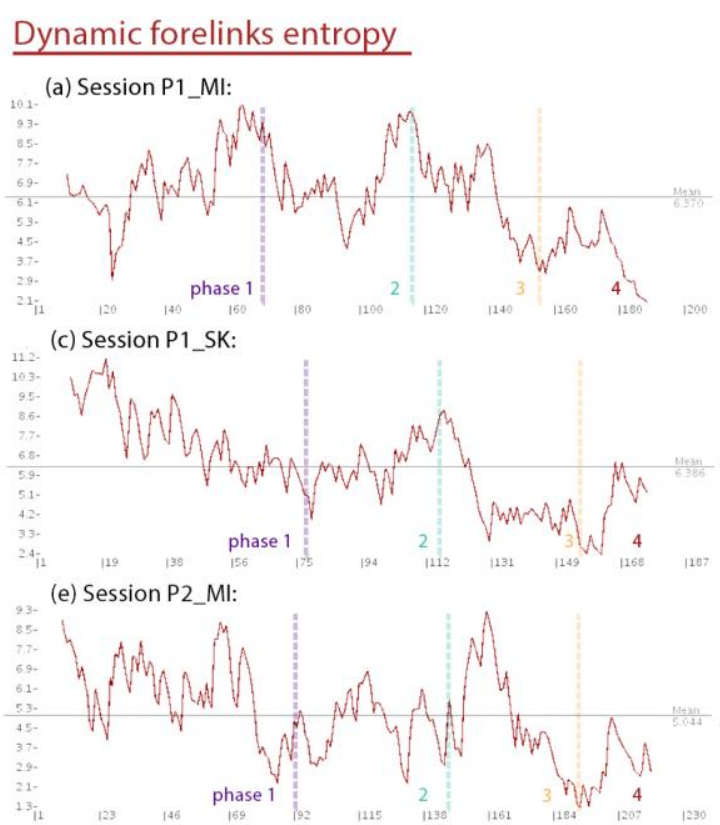

Dynamic backlinks entropy

(b) Session P1_Ml:

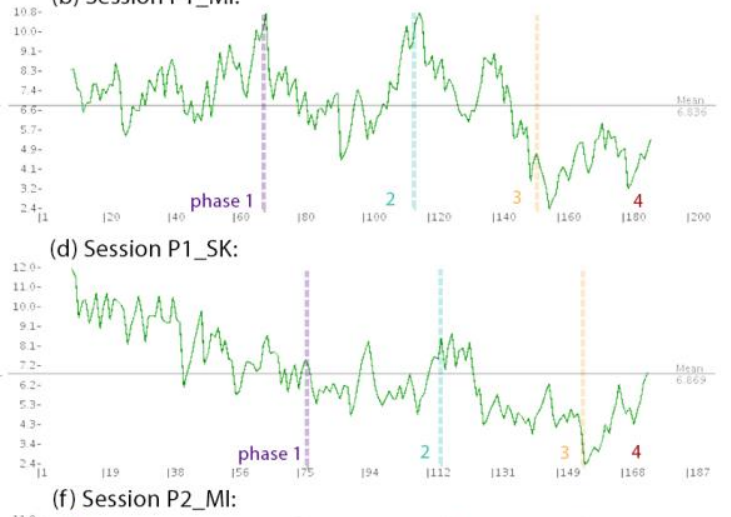

(g) Session P2_SK
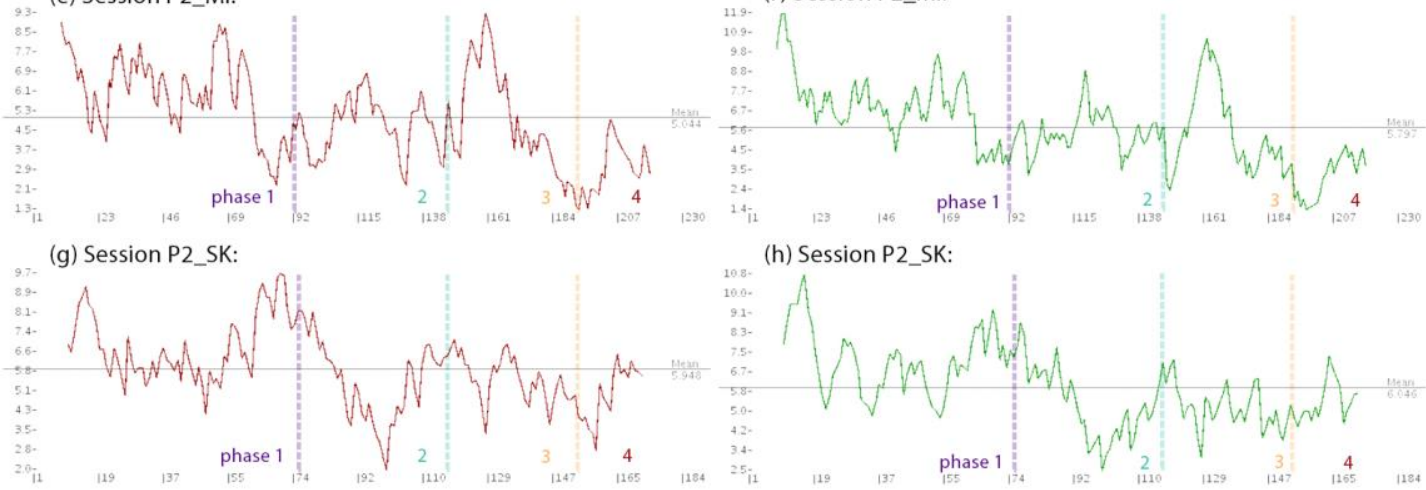

(h) Session P2_SK:

(i) Session P3 MI:

phase $1 \underbrace{52.0}_{173}$
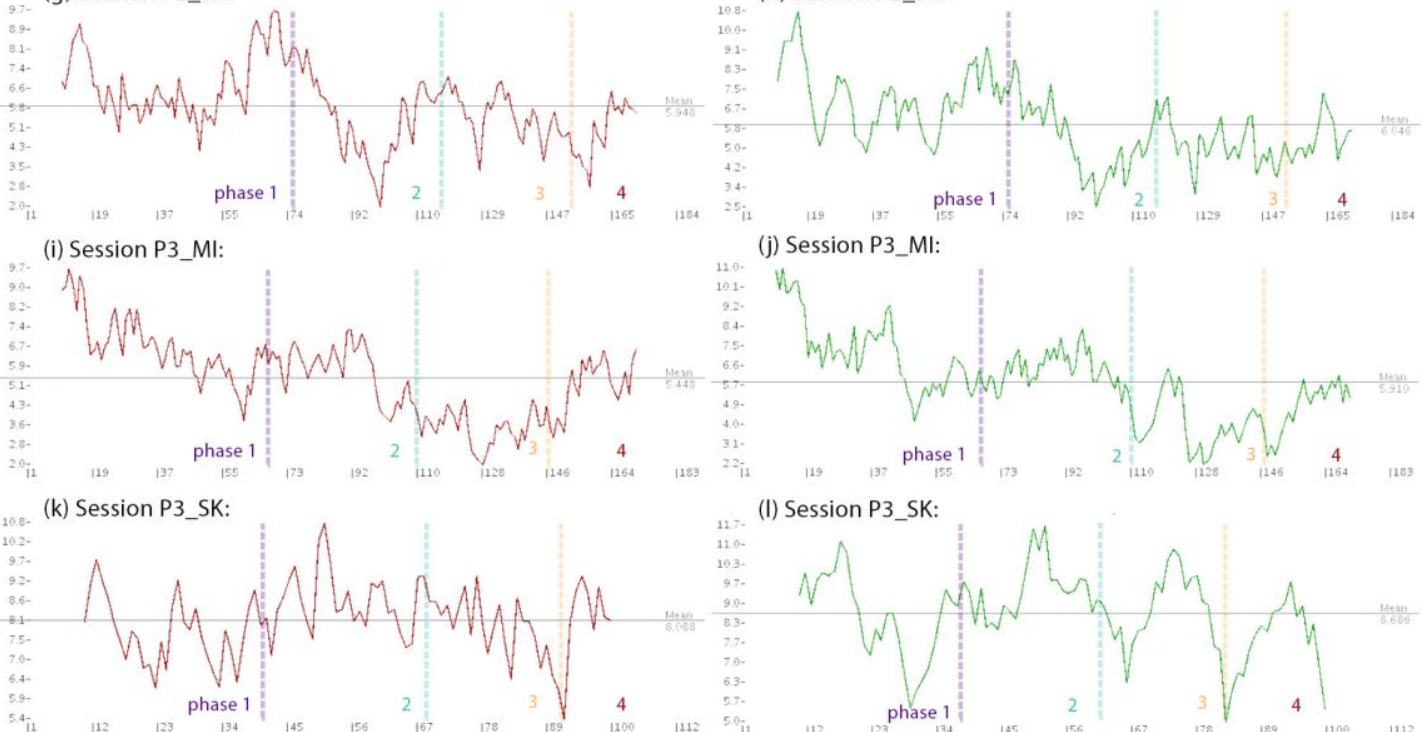
(j) Session P3_MI

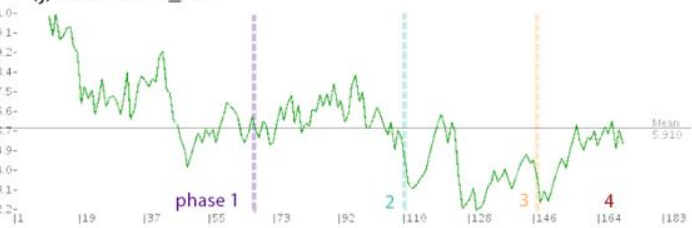

(I) Session P3_SK:

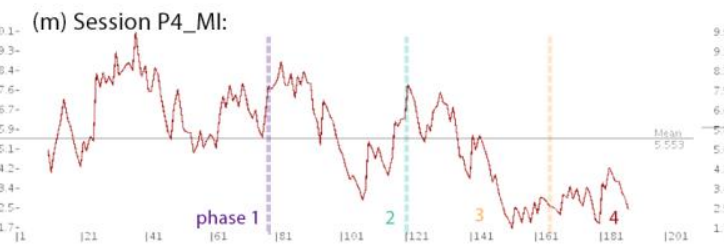

(o) Session P4_SK:

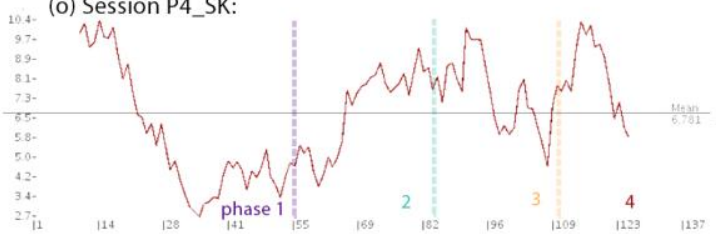

(p) Session P4_SK:
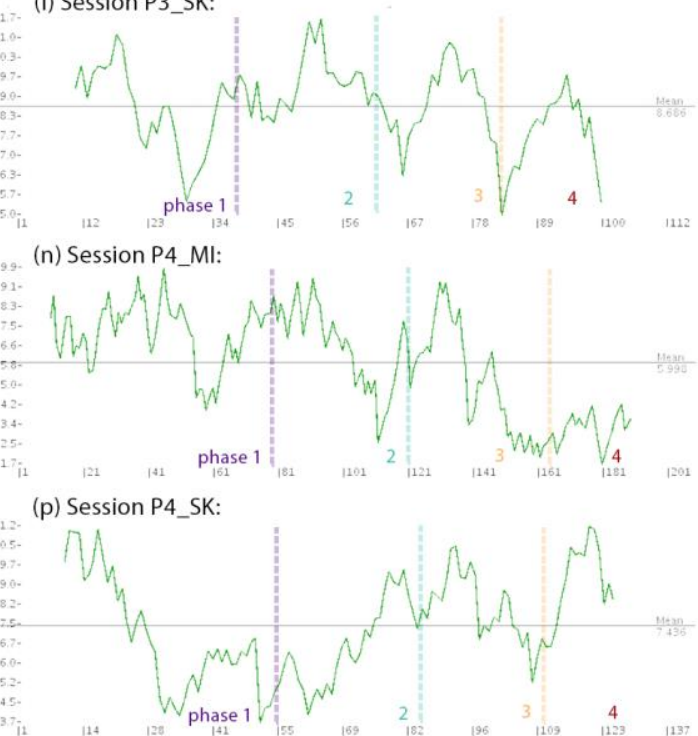

Figure 5. Dynamic forelinks and backlinks entropies of eight sessions 
Figure 5 elicits forelink and backlink evolution throughout eight design sessions. While forward links illustrate acts of ideation (initiations), backward links to what has already been done make sure that a good fit exists, illustrating the act of evaluation (enhancements and responses). With linkograph entropy, linked segments and distribution of links can be inferred. It shows how a rich idea generation process is indicated by: well- integrated structure of ideas and variety of moves. The entropy and productivity of design activities are considered to be related (Kan, Bilda, and Gero 2007). With the exception of P3_MI and P3_SK sessions, in each session, forelink and backlink entropies follow close to similar patterns in terms of significant peaks and dips.

\begin{tabular}{|c|c|c|c|c|c|}
\hline Participant & Design sessions & Highest CMs> & Move no & Highest $<$ CMs & Move no \\
\hline \multirow[t]{2}{*}{$\mathrm{P} 1$} & P1_MI & $\mathrm{CM}^{8}>$ & \#3, \#37 & $<\mathrm{CM}^{5}$ & $\# 127$ \\
\hline & P1_SK & $\mathrm{CM}^{13}>$ & $\# 33$ & $<\mathrm{CM}^{8}$ & $\# 145$ \\
\hline \multirow[t]{2}{*}{$\mathrm{P} 2$} & P2_MI & $\mathrm{CM}^{13}>$ & $\# 40$ & $<\mathrm{CM}^{7}$ & $\# 63$ \\
\hline & P2_SK & $\mathrm{CM}^{10}>$ & $\# 62$ & $<\mathrm{CM}^{6}$ & $\# 128, \# 151$ \\
\hline \multirow[t]{2}{*}{ P3 } & P3_MI & $\mathrm{CM}^{10}>$ & $\# 8$ & $<\mathrm{CM}^{6}$ & $\# 98, \# 127$ \\
\hline & P3_SK & $\mathrm{CM}^{9}>$ & $\# 10$ & $<\mathrm{CM}^{6}$ & \#22, \#31, \#77 \\
\hline \multirow[t]{2}{*}{ P4 } & P4_MI & $\mathrm{CM}^{9}>$ & \#44 & $<\mathrm{CM}^{7}$ & $\# 188$ \\
\hline & P4_SK & $\mathrm{CM}^{9}>$ & $\# 9$ & $<\mathrm{CM}^{6}$ & $\# 80$ \\
\hline
\end{tabular}

Table 6. Highest CM (Critical Moves) in eight sessions

Critical moves are particularly important in design process as they show the interlinking processes of moves.Table 6 depicts critical moves in each session, with a tendency that critical moves due to forelinks $(\mathrm{CMs}>)$ are higher than critical moves due to backlinks $(<\mathrm{CMs})$. Early indication reveals that the ideation rate in all sessions is higher compared to the evaluation rate.

\section{Discussions}

Outline of this section will be based on two hypotheses posited in the Introduction. Relevant data will be presented (referred back from results section) according to the 
specific questions and where necessary additional data will also be demonstrated.

Within each participant, the use of different environments to generate ideas could possibly substitute each other. In terms of difference of link indices (Table 3), P3 posited the highest. P3_MI's link index was 44.44\% higher than P3_SK session. Whilst P1 secured 29.68\% in favour of P1_SK session, P2 had 11.32\% in favour of P2_MI session and P4 had 6.72\% in favour of P4_SK session. An early indication shows that both environments are equally significant, for the given duration of time.

It has been established that both sketches and mental imagery support ideation in unique ways. An atypical feature of sketches are they allow designers to engage in broad design strategies, across different levels of detail and attention. The repetitiveness of sketches also facilitates seeing and interpreting, through (but not limited to) the act of drawing and re-drawing. Mental imagery supports design ideation in different ways. The ease and speed are useful to generate quick ideas or simulating options. In P3_MI session, three ideas were investigated in approximately 10 minutes (moves \#54, \#66 and \#86), in comparison with the P3_SK session, with 19 minutes spent to develop three distinctive ideas (\#51-\#64, \#66-77, \#78-94).

The nature of interplay in SK sessions and MI sessions are different in that coexistence between internal and external representations in MI sessions, happened during 10 minutes at the end of sessions. The transition between ideas generated in BF sessions (the first 35 minutes) and how they were externalised is also within the scope of this paper. This first notion relates to Hypothesis 1. In SK sessions, they were exhibited during the whole session for 45 minutes (related to Hypothesis 2).

\section{H1- In sessions which access to external representations are limited, mental images are recalled to be manipulated further during EXT session.}

This hypothesis will be investigated by examining: how mental images generated during 
BF (Blindfolded) sessions were externalised during EXT (Externalisation) sessions; when access to externalisations during BF sessions was withdrawn, how designers carried on with their designing activities; and lastly the period where design activity rates dropped where externalisations were not possible.

(a) Session P1_MI (EXT session only):

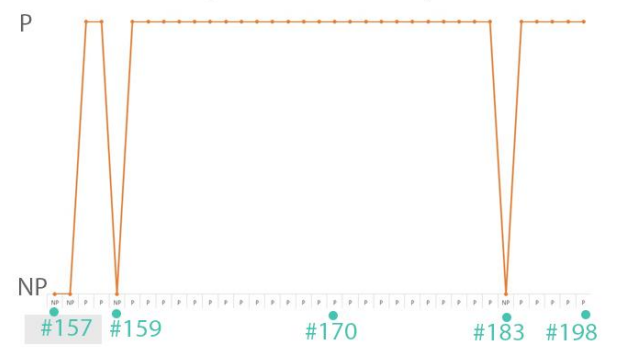

(c) Session P3_MI (EXT session only):

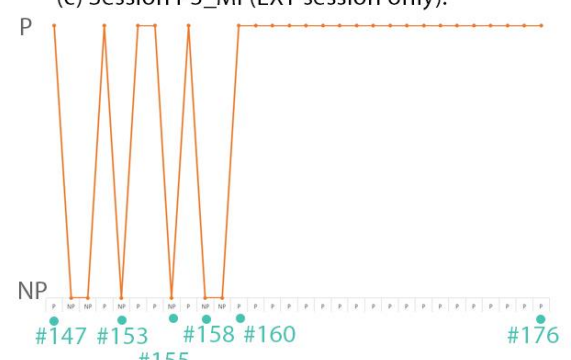

(b) Session P2_MI (EXT session only):

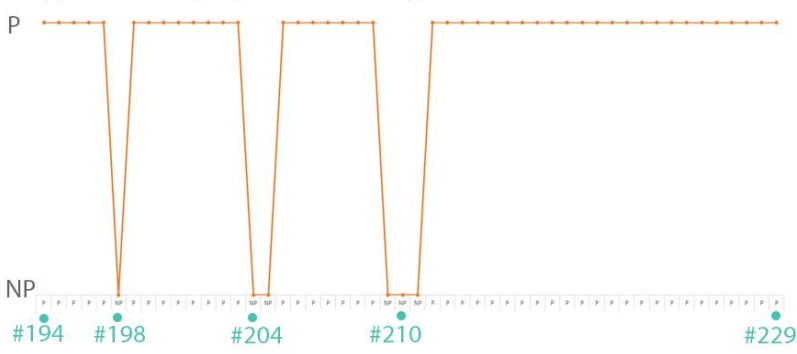

(d) Session P4_MI (EXT session only):

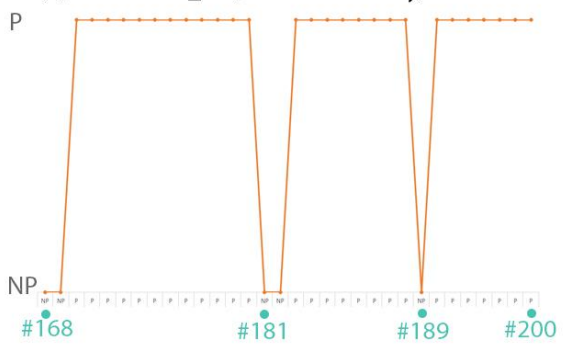

LEGEND: NP: non-physical activities P: physical activities

Figure 6. Types of manifestation mental images were recalled

Firstly, it was expected that mental images generated and manipulated during BF sessions (Phase 1- 3) would be externalised through sketches in EXT sessions (Phase 4). From the linkographs (Figure 4), P1 and P4 referred back to previously mentioned ideas as far back as the first half of phase 1 including the first few moves, whilst P2 and P3 referred as far back as the first half of phase 2. The recalling processes involved physical activities (drawings mostly, and also texts) and non-physical activities (cognitive actions only which were inferred by what participants talked out loud about), refer to Figure 6.

Table 7 shows percentage of moves which are linked from EXT session where designers externalise their mental images, from BF sessions. An average of $58.32 \%$ of total moves in EXT sessions (Phase 4) were linked to Phase 1 to Phase 3. It suggests a 
pertinent question about additional design manipulation activities during the EXT session (Phase 4). In the case of P1, backlinks to Phase 1- Phase 3 occurred, while at the same time, the same moves have forelinks within Phase 4 (see P1_MI move \#181 in Figure 7, highlighted in orange); similarly with P2 and P4. It seems that after thoughts were recalled, they were developed further.

\begin{tabular}{|l|lll|l|l|}
\hline Participant & Total moves in & Number of unlinked & \% of unlinked moves & \% of linked moves \\
\hline P1 & EXT session & moves to BF session & to BF session & to BF session \\
\hline P2 & 44 & 17 & 38.64 & 61.36 \\
\hline P3 & 37 & 12 & 32.43 & 67.57 \\
\hline P4 & 36 & 19 & 52.78 & 47.22 \\
\hline & 35 & 15 & 42.86 & 57.14 \\
\hline
\end{tabular}

Table 7. Linked and unlinked moves from EXT to BF session

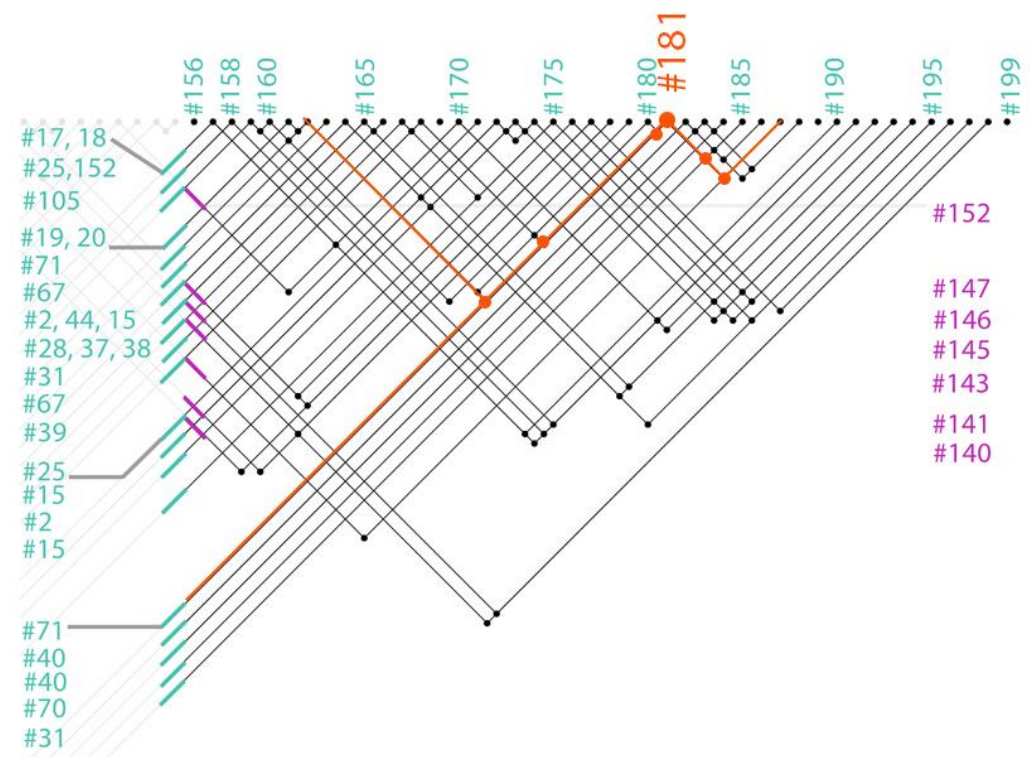

Figure 7. Sliced EXT sessions' linkographs of P1_MI session

The interplaying roles between internal and external representations in EXT sessions are not simply generative (externalising previously envisaged internal representations), but also provocative to idea exploration in a way that, once information was recalled, designers manifested them into drawings or texts to be manipulated further. Sole cognitive actions appeared sparsely when designers tried to 
explain about the ideas or concept (for instance in P3_MI session) or justifying what they were drawing (in P4_MI session). In summary, mental images could be recalled in the form of drawings, texts or sole cognitive actions (without physical movements). Through a process of reformulation of structure variables and analysis; there was a possibility that design was then manipulated further during this 10 minute EXT session.

Secondly, from two functions of gesture mentioned in the literature review section (Visser 2009), the authors seek to identify the first function: offering possibilities to render spatial and motion-related qualities of design objects through simulation. Coded gesture in Figure 8 illustrates prominent gestures made during BF sessions when designers did not have access to visual input while thinking out loud. Whilst P1 tend to use gesture less than the other three participants, P2 showed hand gestures often. P3's bodily movement was often rocking back and forth. P4 used hand gestures to emphasise their statements.

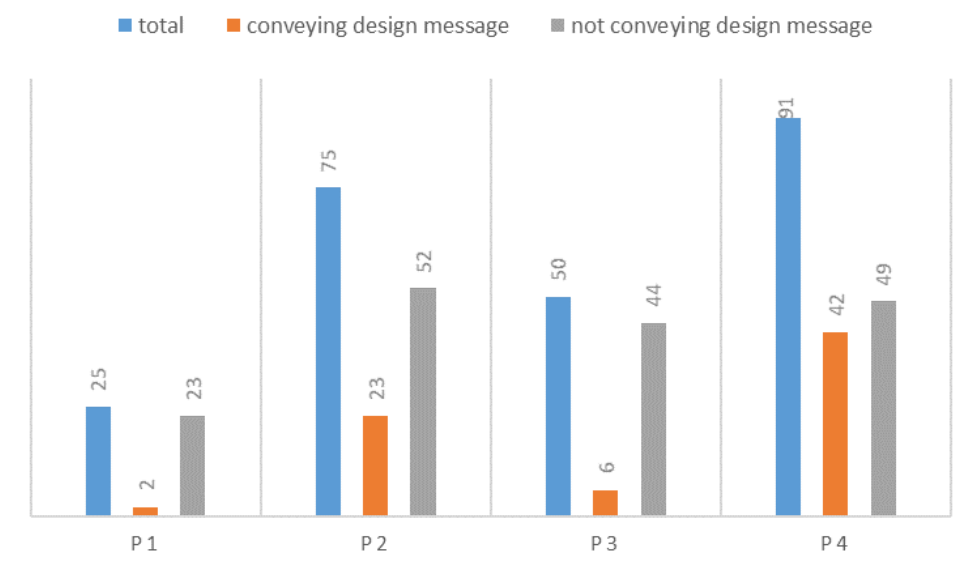

Figure 8. Gesture used in BF sessions

In terms of gestures which convey design message, some observed functions will be posited along with an example. They were: 1) dimension thinking, 2) spatial reference, 3) emergent ideas, 4) simulation tool, 5) shapes and 6) sketching-like movement. The first function is dimension related. For instance during P2_MI session, hand gesture was used to make the shape of a staircase while calculating mentally. An 
example of the second function, 'surrounding area' was illustrated by doing a circle using finger movement on the table and making space inference from in between two frames by simulating two frames with both hands (P4_MI session). It appeared that gesture might aid to illustrate spatial quality and spatial components. The third function was noticed at the beginning of ideas or new concepts. This might relate to the refocussing role of gesture, it aids thinking about something different from previous moves. The fourth function, gesture was used as a simulation tool. It could be motionrelated quality (P1_MI session, making a 'sliding' movement with the index finger), spatial render (imagining that the subject was standing on a tall building in P3_MI session) or others such as rendering colour and finishes (P4_MI session). The fifth function, gesture, has been used to express curved shapes (P2_MI session) by making curves using fingers and rectangles using two hands (P4_MI session). Sixth was sketching-like movement where designers made hand gestures as if they were sketching. An example, P4_MI session was referring to "frame" when miming sketching without pen and paper for 20 seconds.

In terms of gesture which did not convey design message, they were bodily movement to adjust sitting posture or referring to themselves (for instance, $\mathrm{P} 2$ often did this). It seems with changing posture, in some cases it helped the designer to re-focus.

Thirdly, at the end of Phase 2 (after 25 minutes) with the exception of P3, forelink entropy had a decreasing pattern and the lowest points were in Phase 3. In terms of backlink entropy, after 25 minutes and during Phase 3, all four MI sessions started with a drop and subsequently a significant rise. This might suggest that between 25-35 minutes in MI sessions, idea generation started to slow down and cognitive activities start to decrease. This might also explain the two distinct chunks, refer to Figure 3(a,c, e and g), split approximately in the middle of BF sessions. It is in 
accordance with previous findings that after 20 minutes, cognitive and perceptual activity in design sessions without access to externalisations dropped below sessions with access to externalisations (Bilda, Gero, and Purcell 2006).

\section{H2- With an access to external representations, the interplay between sketches} and mental imagery vary in terms of sequence and reciprocity, depending on designers' strategy and intention.

The hypothesis will be expanded by looking at an excerpt illustrating how sketches and mental imagery intertwine with each other; and a more detailed investigation about the mechanical aspect of the interplay.

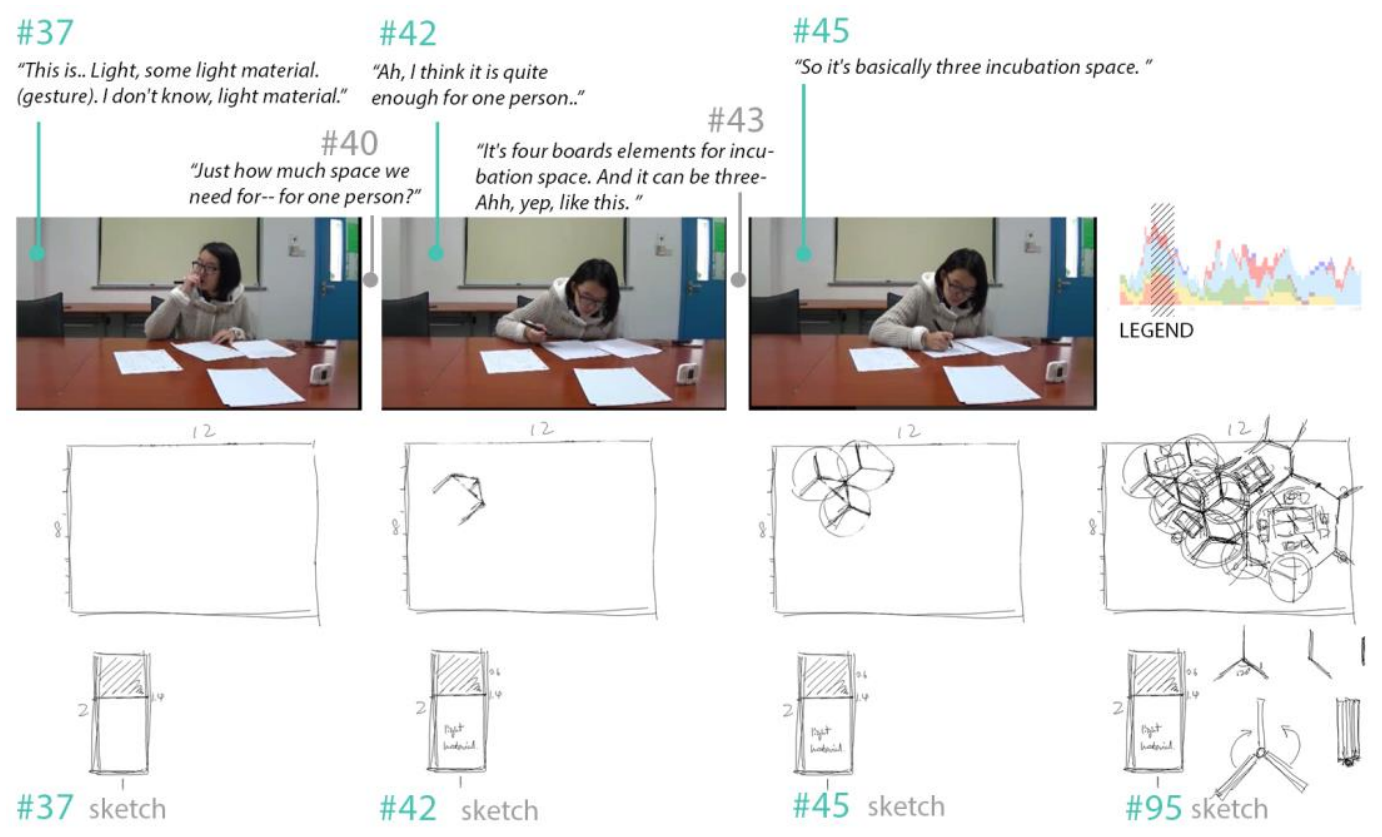

Figure 9. Excerpt of P1_SK session, utterances \#37-\#95

First of all, the nature of interplay in SK sessions is illustrated in Figure 9. It shows an excerpt of P1_SK session around utterance \#37 which was one of the critical moves according to Table 6 . In move \#37, P1 was thinking about a $2 \mathrm{mx} 1 \mathrm{~m}$ modular component. The shaded part indicates the transparent material and the non-shaded one use of a light material, which was annotated in move \#42. In move \#37, the participant looked away out of the window and mentioned about 1.4 meters of height and light 
material. Mental images were active parts of thinking and sketching, and from this particular move P1 imagined the height using the room's physical setting as a reference point.

From the excerpt, it can be seen that the interplaying roles between the two in SK sessions are possibly: supporting role (the feedback loop between physical and nonphysical properties), co-evolving role (both evolve at the same time) and reflecting roles (for instance, how both take turns to aid inspecting processes).

Secondly, concepts of simultaneity between internal representations and external representations will be explored. In visual processing during mental imagery, four types of functions on which imagery rely (Kosslyn, Thompson, and Ganis 2006) are the point of departure of this inquiry. Kosslyn identified these functions in the course of mimicking imagery when building a computer simulation and justified by the effect of damage in imagery and neuro-imagining studies (ibid). The four types are: 1) image generation (IG, henceforth) 2) image inspection (II, henceforth) 3) image maintenance (IM, henceforth) and 4) image transformation (IT, henceforth).

Parts of four SK sessions were used to illustrate. Moves were selected around the highest peak based on semantic dynamic processes (Figure 3) and critical moves (Table 6). Ten utterances around the peaks were coded based on IG, II, IM and IT; for both visual processing during 1) mental imagery and 2) during the use of pen-andpaper, looking at sketching behaviour. Figure 10 illustrates the two concurrent processes in a selected five- move chunk, the sequence is colour coded. When there is more than one process in one move, arrows are used to clarify the sequence.

Five notions can be identified. Firstly, external representations are utilised to inspect an idea concurrently. During P1_SK session, move \#32; P1 was using the grid 
on the paper to count and proposed an area of $8 \times 12$ meter. P1 generated the footprint and at the same time inspected whether 8 meters and 12 meters would be sufficient.

(a) P1_SK. Move \#32-36:

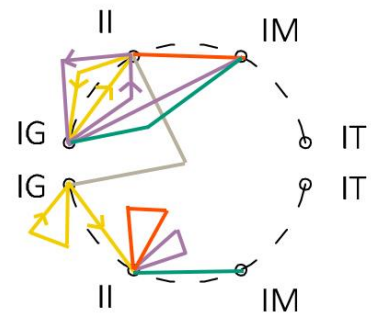

(c) P2_SK. Move \#114-118:

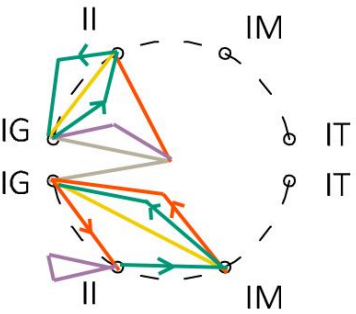

(e) P3_SK. Move \#15-19

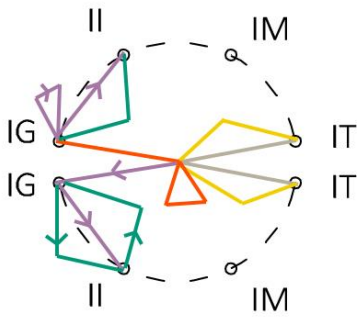

(g) P4_SK. Move \#14-18
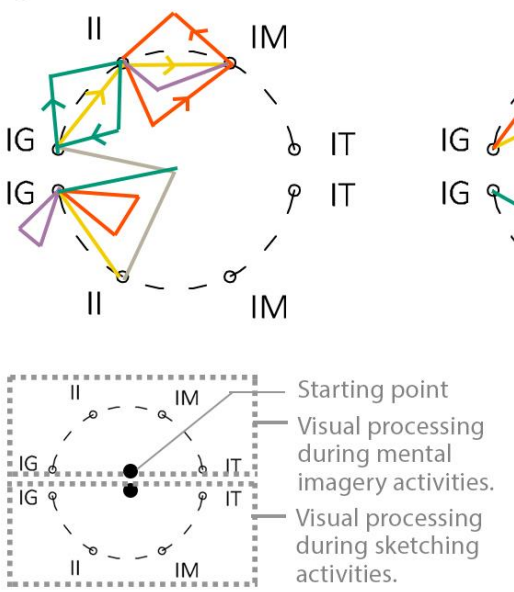

SEQUENCE:

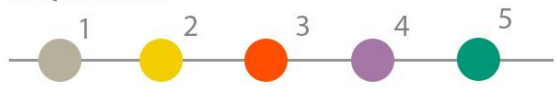

IG

IG

(b) P1_SK. Move \#37-41:

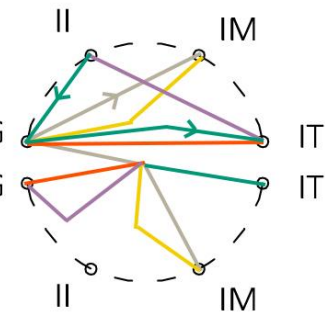

(d) P2_SK. Move \#1 19-124:

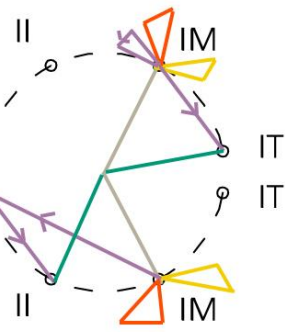

(f) P3_SK. Move \#20-24:

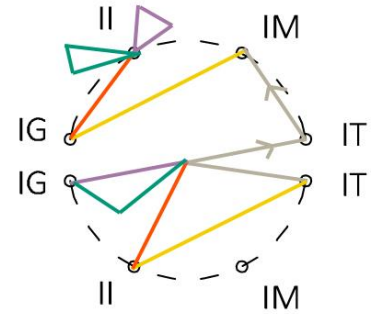

(h) P4_SK. Move \#19-24:

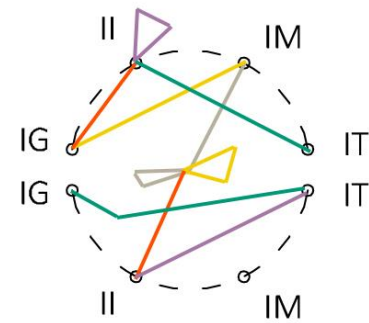

LEGEND:

IG : IMAGE GENERATION

II : IMAGE INSPECTION

IM : IMAGE MAINTENANCE

IT : IMAGE TRANSFORMATION

$>$ : MORE THAN ONE PROCESS

IN ONE MOVE TO SHOW

SEQUENCE

Figure 10. Visual processing of selected moves 


\begin{tabular}{|l|l|l|l|}
\hline Session & Move no & In MI activities & In SK activities \\
\hline P1_SK & $\# 35$ & Generate-Inspect-Generate & Inspect \\
\hline P1_SK & $\# 37$ & Generate-Maintain & Maintain \\
\hline P1_SK & $\# 38$ & Generate & N/a \\
\hline P3_SK & $\# 17$ & Generate & \\
\hline
\end{tabular}

Table 8. Excerpt of P1 and P3 sessions

Secondly, related to the generative role of mental imagery, external representations aided designers to generate ideas using mental imagery. Table 8 shows an excerpt of individual moves and how external representations provoked idea generation in two possible ways: 1) with a feedback loop (for instance, generateinspect-generate) within one move or 2) without a feedback loop- for instance, the second example in Table 8.

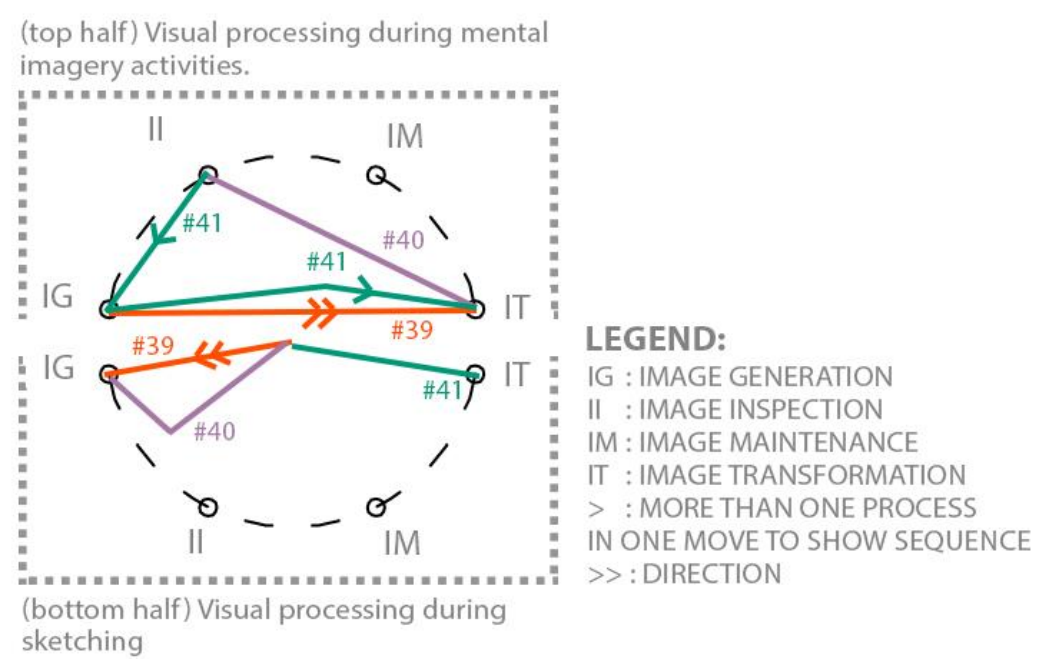

Figure 11. P1_SK session, move \#39-41

Thirdly, in terms of the transformative role of mental imagery; when a mental image was transformed, it needed to be externalised. At the upper part of Figure 11 (refer to orange lines), visual processing during mental imagery showed that after an image was generated (IG), in move \#39, it was transformed. In SK (the lower part), a physical image was generated. Subsequently in \#40, in the upper part, the image was inspected, whilst there is no drawing activity. At this point, P1 was staring at the 
drawings while inspecting. In move \#41, in the upper part, a new idea was generated (after it was transformed and inspected in the previous two moves). This was when P1 decided to modify the shape of a modular 3 boards to 4 boards. Concurrently, a transformed sketch was drawn.

Fourthly, in opposition to the last two examples in Table 8, where there were no sketching activities or image generation using mental imagery; it was noticed that it might be vice versa. When visual processing in mental imagery was on hold (nil), designers generated images using sketches, before the next image generation took place (P2_SK session, move \#116 and \#117). P2 generated and inspected the drawings, and subsequently generated an image using mental imagery in the next move.

Fifthly, within one move, it was possible that there were two chunks of operations instead of two intertwined with each other; for example, processing using mental imagery first, then using drawings subsequently. In P2_SK session, move \#123, P2 looked at the previous drawing (plan) and mentally transformed the plan to isometric drawing. The mental image was maintained by looking at the previous drawing, performing the transformation of view mentally; and lastly generating the isometry sketch and inspecting the possibility of having bookshelves while drawing.

To summarise from the early indications, the way the two representations are intertwined are unique and which strategy to be deployed depends on the designers' intention. They shared mutual interaction, affecting each other reciprocally and these events did not exist in isolation. Also, the interplay can happen at the exact same moment or it can be a distant simultaneity. 


\section{Conclusions and recommendations}

\section{"What are the interplaying roles of sketches and mental imagery in the idea generation stage?"}

With access to external representations during SK (sketching) sessions; they are supporting role, co-evolving role and reflecting role. With access to external representations after $(\mathrm{BF})$ blindfolded session; it is a generative role in terms of externalising previously thought internal representations, but also can be provocative to idea exploration in the way that recalled thoughts can lead to more design development. In terms of simultaneousness, it appears that external and internal representations do not always coexist at the exact time. Sometimes an action precedes the other, but what is certain is that they mutually interact.

\section{H1- In sessions which access to external representations are limited, mental images are recalled to be manipulated further during EXT session.}

The nature of interplay in MI (mental imagery sessions) were expected as a forced offloading of activities by documenting the final proposal through sketches; although there is a possibility that design was then manipulated further during this 10 minutes EXT session. When access to externalisations were withdrawn during BF (blindfolded) sessions, gesture was prominently used but not to the extent that it substituted the use of external representations such as drawings. In terms of recalling mental images, it can be in the form of physical activities (with drawings or texts) or non-physical activities (sole cognitive actions) through a process of reformulation of structure variables and analysis. 


\section{H2- With an access to external representations, the interplay between sketches and mental imagery vary in terms of sequence and reciprocity, depending on designers' strategy and intention.}

In SK sessions, mental imagery was the active part of sketching activities. From five different notions observed in discussions, it is concluded that between internal and external representations; there is shared mutual interaction. They both affect each other reciprocally. The sequence and which one affects which within the sequence are highly dependent on designers' strategy and perhaps the phase in which designers currently are; and it can happen at the exact same moment or distantly. It is postulated that there are more typical conversations between sketches and mental imagery to be explored. The next task will be to identify a formal description of designers' strategies and how does the interplay differ in each intention.

With regards to previous findings (Table 1), in studies of expert designers: looking at the comparison of link indices between two sessions, there is a strong indication that novice designers are as capable as experts at using mental imagery only. The authors however stipulate that ways of manipulating images are different in expert and novice designers during BF sessions, in that simulation is needed at all times. Moreover, during EXT sessions, novice designers seemed to recall the basic idea manipulated during BF sessions to be further analysed. This observation might be in accordance with Kavakli and Gero (2001), who suggested that expert's ability to use remembered information is the key to the difference. It is however not the scope of this paper and comparison between expert and novice designers needs to be conducted.

Based on these preliminary findings, it is hoped that more attention towards the use of mental imagery in design education might suggest a new pedagogical approach. In terms of design practice, the study is hoped to contribute to similar studies of expert 
designers. Challenges of the study are noted in terms of coded protocols. In future work, for clearer and more distinguishable exploration of the concept of simultaneity, sketching and mental imagery behaviour should be very distinctive and coded separately. In addition, with a limited number of design sessions, this paper cannot generalise the findings. However, it provides an early indication pertaining to the interplay in novice designers. It also yields a starting point for a larger scale of study.

\section{References}

Anderson, R.E., and T. Helstrup. 1993. "Multiple perspectives on discovery and creativity in mind and on paper." In Imagery, creativity and discovery: a cognitive perspective, edited by M J Instons-Peterson B Roskos-Ewoldsen, R E Anderson, 223-53. Amsterdam: Elsevier Science.

Athavankar, U. A. 1997. "Mental imagery as a design tool." Cybernetics and Systems 28 (1):25-41. doi: 10.1080/019697297126236.

Athavankar, U., and A. Mukherjee. 2003. Blindfolded classroom: Getting design students to use mental imagery. Edited by U. Lindemann, Human Behaviour in Design: Individuals, Teams, Tools.

Bilda, Zafer, and John S. Gero. 2004. "Analysis Of A Blindfolded Architect's Design Session." In, 121-36. University of Sydney: Key Centre of Design Computing and Cognition.

Bilda, Zafer, and John S. Gero. 2006. "Reasoning with internal and external representations: A case study with expert architects." In The Annual Meeting of Cognitive Science Society, edited by R. Sun, 1020-6. Lawrence Erlbaum Associates.

Bilda, Zafer, and John S. Gero. 2007. "The impact of working memory limitations on the design process during conceptualization." Design Studies 28 (4):343-67. doi: http://dx.doi.org/10.1016/j.destud.2007.02.005.

Bilda, Zafer, and John S. Gero. 2008. "Idea Development Can Occur Using Imagery Only." In Design Computing and Cognition '08: Proceedings of the Third International Conference on Design Computing and Cognition, edited by Zafer Bilda and John S. Gero, 303-20. Dordrecht: Springer Netherlands.

Bilda, Zafer, John S. Gero, and Terry Purcell. 2006. "To sketch or not to sketch? That is the question." Design Studies 27 (5):587-613. doi: http://dx.doi.org/10.1016/j.destud.2006.02.002.

Christensen, Bo T., and Christian D. Schunn. 2009. "The role and impact of mental simulation in design." Applied Cognitive Psychology 23 (3):327-44. doi: 10.1002/acp.1464.

Ericsson, K.A., and H.A. Simon. 1993. Protocol Analysis: Verbal Reports as Data Revised Edition. Cambridge, Mass: MIT Press.

Finke, R. A., T.B. Ward, and S.M. Smith. 1992. Creative Cognition: Theory, Research and Applications. Cambridge: MIT Press.

Fish, J., and S. Scrivener. 1990. "Amplifying the Mind's Eye: Sketching and Visual Cognition." Leonardo 23 (1):117-26. 
Gero, J. 1990. "Design Prototypes: A Knowledge Representation Schema for Design " AI Magazine 11:26.

Gero, John S., and Udo Kannengiesser. 2004. "The situated function-behaviourstructure framework." Design Studies 25 (4):373-91. doi: http://dx.doi.org/10.1016/j.destud.2003.10.010.

Gero, John S., and Thomas Mc Neill. 1998. "An approach to the analysis of design protocols." Design Studies 19 (1):21-61. doi: http://dx.doi.org/10.1016/S0142694X(97)00015-X.

Goldschmidt, Gabriela. 2013. "How I Became a Design Researcher." In ICoRD'13, edited by Amaresh Chakrabarti and Raghu V. Prakash, 3-13. Springer India.

Goldschmidt, Gabriela. 2014. Linkography : unfolding the design process, Design thinking, design theory: MIT Press.

Jammer, Max. 2006. Concepts of simultaneity [electronic resource] : from antiquity to Einstein and beyond / Max Jammer: Johns Hopkins University Press.

Kan, Jeff W. T., Zafer Bilda, and John S. Gero. 2007. "Comparing entropy measures of idea links in design protocols: Linkography entropy measurement and analysis of differently conditioned design sessions." Ai Edam-Artificial Intelligence for Engineering Design Analysis and Manufacturing 21 (4):367-77. doi: 10.1017/s0890060407000339.

Kavakli, Manolya, and John S. Gero. 2001. "Sketching as mental imagery processing." Design Studies 22 (4):347-64. doi: http://dx.doi.org/10.1016/S0142694X(01)00002-3.

Kavakli, Manolya, and John S. Gero. 2002. "The structure of concurrent cognitive actions: a case study on novice and expert designers." Design Studies 23 (1):2540. doi: http://dx.doi.org/10.1016/S0142-694X(01)00021-7.

Kokotovich, Vasilije, and Terry Purcell. 2000. "Mental synthesis and creativity in design: an experimental examination." Design Studies 21 (5):437-49. doi: http://dx.doi.org/10.1016/S0142-694X(00)00017-X.

Kosslyn, S. M., G. Ganis, and W.L. Thompson. 2006. Mental Imagery and the Human Brain. Paper presented at the 28th International Congress of Psychology, Beijing.

Kosslyn, S. M., W. L. Thompson, and G. Ganis. 2006. The Case for Mental Imagery, Oxford Psychology Series: Oxford University Press USA.

Pourmohamadi Mahounaki, Morteza, and John S. Gero. 2011. LINKOgrapher: An analysis tool to study design protocols based on FBS coding scheme. Paper presented at the 18th International Conference on Engineering Design: Impacting Society through Engineering Design, Technical University of Denmark.

Singh, A. 1999. "The Potential of Mental Imaging in the Architectural Design Process." In IDATER 1999 Conference, 230-6. Loughborough: Loughborough University.

Trafton, J. Gregory, Susan B. Trickett, and Farilee E. Mintz. 2005. "Connecting Internal and External Representations: Spatial Transformations of Scientific Visualizations." Foundations of Science 10 (1):89-106. doi: 10.1007/s10699005-3007-4.

Verstijnen, I. M., C. van Leeuwen, G. Goldschmidt, R. Hamel, and J. M. Hennessey. 1998. "Sketching and creative discovery." Design Studies 19 (4):519-46. doi: http://dx.doi.org/10.1016/S0142-694X(98)00017-9.

Visser, Willemien. 2009. "The function of gesture in an architectural design meeting." arXiv preprint arXiv:0908.3362. 